060.

\section{Safety of Nonphysician Aeromedical Crew Administration of Fentanyl for Prehospital Trauma Analgesia}

Stephen H. Thomas, $M D,{ }^{*}{ }^{1,2}$ William Benevelli, EMT-P, ${ }^{1}$ David Brown, MD, ${ }^{2}$ Suzanne K. Wedel ${ }^{1,3}$

1. Boston MedFlight, Boston, Massachusetts USA

2. Department of Emergency Medicine, Massachusetts General Hospital/Harvard Medical School, Wellesley, Massachusetts USA

3. Department of Surgery, Boston University School of Medicine, Boston, Massachusetts USA

Purpose: Studies highlighting inadequacy of analgesia administered to acutely injured patients have not addressed pain management in the prehospital setting. The objective of this study was to demonstrate the safety of an air medical service protocol for administration of fentanyl analgesia to victims of trauma.

Methods: Flight records from 1994 for all trauma scene responses by an urban rotor-wing nurse/paramedic air medical service were analyzed to identify patients receiving fentanyl (FENT) for analgesia/sedation. Study parameters were systolic blood pressure (BP), heart rate (HR), respiratory rate (RR), Glasgow Coma Scale (GSC) score, and $\mathrm{O}_{2}$ saturation (SAT). Pre-FENT data were defined as values recorded on the flight record just prior to FENT administration; post-FENT data were defined as the first set of values after fentanyl administration. The $t$-test $(\alpha=0.05)$ was used to compare pre- and post-FENT values.

Results: Fentanyl (25-200 $\mu \mathrm{g})$ was administered 154 times to 99 patients. No cases of immediate or delayed adverse effects were noted on flight-record narratives or on review of flight records' objective data. There were no significant changes in any of the parameters studied (see Table; values represent means \pm standard deviation).

\begin{tabular}{|c|c|c|c|c|c|}
\hline & BP & HR & RR & GCS & SAT \\
\hline pre-FENT & $159 \pm 29$ & $97.0 \pm 21$ & $16.6 \pm 5.0$ & $14.5 \pm 1.5$ & $99.1 \pm 1.8$ \\
\hline post-FENT & $155 \pm 30$ & $94.7 \pm 21$ & $16.3 \pm 4.5$ & $14.5 \pm 1.5$ & $99.4 \pm 1.4$ \\
\hline$p$-value & .25 & .34 & .67 & .87 & .35 \\
\hline
\end{tabular}

Conclusions: Use of fentanyl for analgesia during prehospital care of trauma patients seems safe. Further (prospective) study of fentanyl's safety and efficacy in the prehospital setting is warranted.
014.

\section{Effectiveness of Mechanical Versus Manual Chest Compression in Prehospital Cardiac Arrests}

Edward T. Dickinson, $M D, N R E M T-P$, Vincent $P$. Verdile, $M D$,

Robert M. Schneider, MS, Richard F. Salluzzo, MD

Department of Emergency Medicine, Albany Medical College, Albany, New York USA

Purpose: To compare the effectiveness of mechanical versus manual chest compressions during out-of-hospital cardiac arrests.

Methods: A prospective randomized study of nontraumatic, adult cardiac arrest patients managed in an all ALS suburban EMS system was conducted over a 10 -month period. Patients were randomized to two groups: mechanical chest compressions with mechanical ventilations (MCC) or human chest compressions and mechanical ventilations (HCC). Sequential digital end-tidal $\mathrm{CO}_{2}\left(\mathrm{ETCO}_{2}\right)$ readings were obtained for all patients as an index of cardiac output. Patient age, response time, initial EKG rhythm, scene time, and arrest outcome were identified for each patient.

Results: Twenty patients were entered into the study, 10 in each treatment group. Three patients in the MCC group were excluded, two because of $\mathrm{ETCO}_{2}$ monitor battery failure and one because of extubation. Measurements in the HCC group revealed a decreasing $\mathrm{ETCO}_{2}$ during the arrests in the $80 \%$ (eight of 10 ) of the cases and an increasing $\mathrm{ETCO}_{2}$ in $20 \%$ (two of 10) of the cases. No decrease in $\mathrm{ETCO}_{2}$ was noted during the arrests in the MCC group, with $57 \%$ (four of seven) of the cases showing an increased $\mathbf{E T C O}_{2}$, and $43 \%$ (three of seven) showing a constant $\mathrm{ETCO}_{2}$. The differences between the $\mathrm{ETCO}_{2}$ measurements of the two groups were found to be statistically significant by Student's two-tailed $t$-test analysis $(p<0.04)$. Both groups had a average scene time of 27 minutes. Both groups were similar with regard to response time, patient age and initial cardiac rhythm. One patient in the MCC group was admitted to the hospital and died 48 hours later. No other patients in the study survived.

Conclusions: In our study, MCC was superior to HCC in maintaining cardiac output as measured by $\mathrm{ETCO}_{2}$ in out-of-hospital cardiac arrests. 